\title{
MONTE CARLO ESTIMATION OF THE EFFICIENCY OF THE THREE CENTER AUGER EFFECT*
}

\author{
A. ZAKRZEWSKI \\ Institute of Physics, Polish Academy of Sciences \\ Al. Lotników 32/46, 02-668 Warszawa, Poland
}

In this communication the effect of impurity quenching of the photoluminescence of $\mathrm{ZnS}$ crystals is discussed. An appropriate mechanism of nonradiative recombination is proposed and its efficiency is estimated.

PACS numbers: $72.20 . \mathrm{Jv}$

\section{Introduction}

It is well known that even a small amount of transition metal (TM) impurities can deactivate the photoluminescence of semiconductor-based phosphors. In our previous studies on the $\mathrm{ZnS}$ :Fe crystals it was shown that there is an efficient energy transfer process between donor-acceptor pairs (DAP) and isolated iron centers [1]. Such transfer is due to the three center Auger effect (TCAR), i.e. the cooperative interaction of three bound carriers - two electrons and a hole. These carriers are bound at three different centers: donor, acceptor and a "killer" center (deep iron center or shallow donor). It was shown previously that TCAR leads to nonradiative recombination of the photoexcited DAP. Both transfer and direct DAP radiative transition depend on distances: DAP-killer for the former and the donor-acceptor for the latter. Thus, the TCAR efficiency depends on the donor-acceptor rate, characterized by the lifetime $\tau_{0}^{-1}$, and the donor-killer distance $R$.

In this paper we present the estimation of the TCAR efficiency utilizing both the deterministic and stochastic description of the TCAR process. The deterministic approach is based on the analysis of the phenomenological rate equation, which has usually the following form:

$$
\mathrm{d} n_{i} / \mathrm{d} t=f\left(\langle\beta\rangle, n_{i}, n_{j}, \ldots, n_{k}\right),
$$

where $n_{i}, n_{j}, \ldots, n_{k}$ denote concentration of $i$-th $(j$-th, $\ldots, k$-th) center, respectively, and $\langle\beta\rangle$ is an averaged rate constant.

* This work is supported in part by research grant of the Committee for Scientific Research No. 204769101. 
Stochastic approach utilized master equation

$$
\mathrm{d} P_{i} / \mathrm{d} t=-\sum_{j} W_{i j} P_{i}+\sum_{j} W_{j i} P_{j}
$$

where $P_{i}$ and $P_{j}$ denote a particular state of a system (say a given configuration of occupied donors, acceptors and killer centers) and $W_{i j}$ denotes probability per unit time for "jumping" from $P_{i}$ to $P_{j}$.

The main difficulty in solving master equation stems from the very complicated summation over all possible $W_{i j}$. On the other hand, deterministic approach is free from these difficulties, but it does not contain any information about transfer probability. For instance, based on deterministic approach only it is not possible to distinguish between various energy transfer mechanisms.

\section{Results and discussion}

We shall start our analysis of the TCAR efficiency with the deterministic approach. We utilized here experimental results obtained by means of the photo-ESR technique for $\mathrm{ZnS}: \mathrm{Fe}$ and $\mathrm{ZnS}: \mathrm{Al}$ crystals $[1,2]$. In the former case the killer center was an isolated iron impurity, in the latter a shallow (S) and a deep (D) Al donor. It was shown previously that for $\mathrm{ZnS}$ :Fe crystals the TCAR process can be directly observed experimentally by monitoring the changes of the $\mathrm{Fe}^{3+} \mathrm{ESR}$ signal intensity under illumination which populates DAP's. From such an experiment we estimated the TCAR efficiency and we showed that the reduction of DAP emission due to TCAR was about $10 \%$.

Phenomenological equation describing kinetics of excitation of the ESR $\mathrm{Fe}^{3+}$ signal intensity after turning off the light is following (for small values of $t$ ):

$$
\mathrm{d} n_{\mathrm{Fe}} / \mathrm{d} t=\left\langle\beta_{\mathrm{TCAR}}\right\rangle n_{\mathrm{D}} n_{\mathrm{A}} N_{\mathrm{Fe}},
$$

where $N_{\mathrm{Fe}}$ and $n_{\mathrm{Fe}}$ denote concentration of iron $\mathrm{Fe}^{2+}$ and $\mathrm{Fe}^{3+}$ and $n_{\mathrm{D}}$ and $n_{\mathrm{A}}$ denote concentration of neutral donors and acceptors, respectively.

Experimental results give following values of $\left\langle\beta_{\text {TCAR }}\right\rangle=10^{-32}-10^{-33} \mathrm{~cm}^{6} / \mathrm{s}$. This values of $\left\langle\beta_{\mathrm{TCAR}}\right\rangle$ can be compared with those obtained by Przybylińska and Godlewski for TCAR-type DAP $\Rightarrow$ donors energy transfer: $\left\langle\beta_{\mathrm{TCAR}}^{\mathrm{S}}\right\rangle=10^{-29}$ $10^{-30} \mathrm{~cm}^{6} / \mathrm{s},\left\langle\beta_{\mathrm{TCAR}}^{\mathrm{D}}\right\rangle=10^{-30}-10^{-31} \mathrm{~cm}^{6} / \mathrm{s}$. One can see the following trend of the $\left\langle\beta_{\text {TCAR }}\right\rangle$ values: $\left\langle\beta_{\text {TCAR }}\right\rangle$ is inversely proportional to the localization radius of the electron bound to killer center.

Stochastic approach needs a calculation of the TCAR rate $W(R)(R$ denotes donor-killer distance). Following Suchocki and Langer [3] (and their theoretical analysis of the two center Auger effect) this can be done in a standard perturbation manner, i.e. $W(R)$ is proportional to the squared matrix element: $W(R) \sim|M(R)|^{2}=\left|M_{\mathrm{D}}(R)-M_{\mathrm{Ex}}(R)\right|^{2}$, where $M_{\mathrm{D}}(R)$ and $M_{\mathrm{Ex}}(R)$ denote direct and exchange matrix element of the interelectron repulsion operator between initial and final states. If the direct term dominates in $W(R)$, it can be taken as a power series

$$
W(R)=\tau_{0}^{-1}\left(R_{0}^{\mathrm{DD}} / R\right)^{6}+\tau_{0}^{-1}\left(R_{0}^{\mathrm{DQ}} / R\right)^{8}+\ldots
$$

If the exchange term dominates, $W(R)$ is described as follows:

$$
W(R)=\exp \left[\gamma\left(1-\left(R / R_{0}^{\mathrm{Ex}}\right)\right], \quad \gamma=2 R_{0}^{\mathrm{Ex}} / L .\right.
$$


Here $R_{0}^{i j}$ denotes critical radius for energy transfer, indices $i j$ show the nature of the interaction (dipole-dipole, dipole-quadrupole and exchange), and the parameter $L$ is close to the half of the donor Bohr radius. In the case of multipole interaction, $R_{0}$ can be estimated from optical measurements, and was shown to be $R_{0} \approx 10 \AA$. Similar values were also obtained for higher multipole terms. Knowing $R_{0}$, one can calculate the relative quantum yield $\eta / \eta_{0}=W_{R} /\left(W_{R}+W_{N R}\right)$ and compare it with experimental results. Here $W_{R}$ and $W_{N R}$ denote total DAP radiative and TCAR nonradiative recombination rates. $W_{\mathrm{R}}$ and $W_{\mathrm{NR}}$ were calculated from Monte Carlo simulations by summing over randomly distributed donors, acceptors and iron. We found that multipole interactions are not sufficient for explaining $10 \%$ reduction of the DAP emission.

The underestimation of the TCAR efficiency is most likely due to the dominant role of the exchange interaction. This hypothesis is supported by the previous observation of Suchocki and Langer [3] of the two center Auger effect which quenches manganese intracenter emission. It was shown that the above effect is caused predominantly by an exchange interaction. Including in our calculations exchange interaction we found that the observed DAP emission deactivation can be achieved for $R_{0}^{\mathrm{Ex}} \approx 20-40 \AA$. In a similar way one can also estimate $R_{0}^{\mathrm{Ex}}$ for the case discussed by Przybylińska and Godlewski [2] (i.e. donor as the third center). The results are summarized in Table.

TABLE

Values of parameters characterizing quantum yield of TCAR.

\begin{tabular}{c|c|c|c}
\hline \hline \multirow{2}{*}{ Crystal } & \multicolumn{2}{|c|}{ Critical distance } & \multirow{2}{*}{$\left\langle\beta_{\text {TCAR }}\right\rangle$} \\
\cline { 2 - 3 } & $R_{0}^{\text {DD }}[\AA]$ & $R_{0}^{\text {Ex }}[\AA]$ & {$\left[\mathrm{cm}^{6} / \mathrm{s}\right]$} \\
\hline $\mathrm{ZnS:Cu}, \mathrm{Fe}$ & $6-12$ & $20-40$ & $10^{-32}-10^{-33}$ \\
ZnS:Al & & $50-70$ & $10^{-29}-10^{-32}$
\end{tabular}

\section{References}

[1] A. Zakrzewski, M. Godlewski, Phys. Rev. B 34, 8993 (1986).

[2] H. Przybylińska, M. Gudlewski, Phys. Rev. B 36, 1677 (1987).

[3] A. Suchocki, J.M. Langer, Phys. Rev. B 39, 7905 (1989). 